\title{
Effects of Knudsen diffusivity on the effective reactivity of nanoporous catalyst media
}

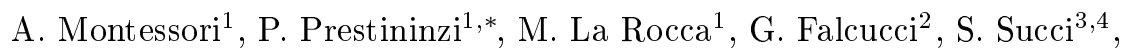 \\ E. Kaxiras ${ }^{4}$
}

\begin{abstract}
We investigate the non-equilibrium hydrodynamic effects on the reactivity of a nanoporous catalytic sample. Numerical simulations using the Lattice Boltzmann Method (LBM) show that non-equilibrium effects enhance the reactivity of the porous sample, in agreement with theoretical predictions [1]. In addition, we provide a quantitative assessment of the reactivity in terms of the thickness of the reactive layer inside the nanoporous catalytic sample. Such an assessment constitutes a first step towards integrated simulations encompassing nanoscale reactivity and transport coefficients within a macroscale description of experimental relevance.
\end{abstract}

\section{Introduction}

The effective control and optimization of catalytic processes is known to play a crucial impact in many fields of applied science and technology, from the synthesis of new materials, to the production of innovative fuels and energy systems of crucial relevance to sustainable industrial growth $[2,3]$. Due to the complex nature of the involved phenomena, characterized by timescales ranging from femtoseconds, $\left(O\left(10^{-15} \mathrm{~s}\right)\right.$, bond breaking/forming events, all the way

\footnotetext{
${ }^{*}$ Corresponding author

Email address: pietro.prestininzi@uniroma3.it (P. Prestininzi)

${ }^{1}$ Department of Engineering, "Roma Tre" University, Via Vito Volterra 62, 00146 Rome, Italy

${ }^{2}$ Dept. of Engineering, University of Naples

${ }^{3}$ Istituto per le Applicazioni del Calcolo, CNR Via dei Taurini 19, 00185 Rome - Italy

${ }^{4}$ Physics Department, Harvard University, Oxford Street, 11, 02145, Cambridge (USA)
} 
up to minutes and hours for reactor operation. Under such conditions, the experimental activity must be assisted by multiscale numerical models aimed at assessing the interplay of the different physical mechanisms involved in the

process. In this paper, we present the results of numerical simulations based on the Lattice Boltzmann Method (LBM), [4, 5, 6, 7, 8, 9, 10, 11, 12, 13, 14, 15, 16], for reactive flows through nano-porous media. LBM has proven to provide a flexible and efficient method for the investigation of fluid dynamic phenomena, especially flows in porous media $[7,17,18,19]$, as well as non-ideal flows at meso- and nano-scales, [20, 21]. When considering passively advected scalars in fluid flows, LBM allows to tune the carrier kinematic viscosity independently from the passive scalar molecular diffusivities. In this paper, we exploit this unique characteristic to investigate flow reactivity in response to variations of molecular diffusivity within non-hydrodynamic regimes.

By resorting to a new reactive boundary condition we assess the influence of characteristic non-dimensional numbers on the overall conversion efficiency of a nano-porous material. In particular, it is found that Knudsen number, a direct measure of departure from hydrodynamic behavior, provides a marginal gain of reactivity. Such gain is found to increase at decreasing diffusive Damköhler number (for definition see Section 3), the ratio of chemical to diffusive timescales, i.e. for materials with relatively low chemical reactivity. Even though our work focuses on general features of the advection-diffusion-reaction problem, it nonetheless provides quantitative insights on the conversion of gold disk-shaped (ingot) nanoporous materials for heterogeneous catalysis applications [22, 23].

\section{The LBM for heterogeneous reactive flows}

The LBM has been described at length in the current literature [24, 25], hence here we only remind the basic aspects most relevant to the present work. The multicomponent LBM can be expressed as follows,

$$
f_{i}^{(k)}\left(\boldsymbol{x}+\boldsymbol{c}_{i}, t+1\right)-f_{i}^{(k)}(\boldsymbol{x}, t)=-\omega\left(f_{i}^{(k)}-f_{i}^{(k) e q}\right)
$$


where $f_{i}^{(k)}$ is the probability density function of finding a particle of the species $k$ at site $\boldsymbol{x}$ at time $t$, moving along the $i-t h$ lattice direction defined by the discrete speeds $\boldsymbol{c}_{\boldsymbol{i}}$, with $i=0, \ldots, b$, where $b=18$ if a three dimensional nineteen speed lattice (D3Q19) is employed. It has been recently shown that a lower order regularized LB model can be used to accurately predict flows in complex porous media even at finite Knudsen numbers [19].

The left hand-side of eq. (1) represents the free-streaming of molecules, whereas the right-hand side accounts for the collisional relaxation towards local Maxwellian equilibrium, on a time-scale $\tau=1 / \omega$. The macroscopic fluid density $\rho$ and velocity $\boldsymbol{u}$ are given by $\rho(\boldsymbol{x}, t)=\sum_{i=0}^{b} f_{i}(\boldsymbol{x}, t)$ and $\rho(\boldsymbol{x}, t) \boldsymbol{u}(\boldsymbol{x}, t)=\sum_{i=0}^{b} \boldsymbol{c}_{\boldsymbol{i}} f_{i}(\boldsymbol{x}, t)$, respectively. The equilibrium distribution function is given by a low-Mach, second-order, expansion of a local Maxwellian, namely:

$$
f_{i}^{k, e q}=w_{i} \rho^{k}\left(1+\frac{1}{c_{s}^{2}} \boldsymbol{c}_{\boldsymbol{i}} \cdot \boldsymbol{u}+\frac{1}{2 c_{s}^{4}}\left(\boldsymbol{c}_{\boldsymbol{i}} \cdot \boldsymbol{u}\right)^{2}-\frac{1}{2 c_{s}^{2}} \boldsymbol{u} \cdot \boldsymbol{u}\right)
$$

In the above, $\boldsymbol{u}$ is the flow velocity of the carrier species. In order to span a wide range of Knudsen number beyond the usual hydrodynamic limits, it proves highly beneficial to filter out non-hydrodynamic modes from the post-collisional distribution $f_{i}^{\prime} \equiv(1-\omega) f_{i}+\omega f_{i}^{e q}$. The procedure, known as "regularized" LBM $[26,27]$, is briefly recalled hereafter. The main idea is to introduce a set of pre-collision distribution functions which are defined only in terms of the macroscopic hydrodynamic moments. All the higher-order non-equilibrium information, often referred to as ghosts [4], is discarded. In equations, the regularized LB reads as follows:

$$
f_{i}\left(x_{i}+c_{i} \Delta t, t+\Delta t\right)=\mathcal{R} f_{i}^{\prime}(x, t) \equiv h_{i}^{e q}-\frac{\Delta t}{\tau} h_{i}^{n e q}
$$

where $h_{i}$ is the hydrodynamic component of the full distribution $f_{i}$ (see $[28,27$, $29,30])$ and $\mathcal{R}$ is the regularization operator. The above equation show that the post-collision distribution, of a $4^{\text {th }}$-order isotropic lattice, is defined only in terms of the conserved and the transport hydrodynamic modes, namely density $\rho$, current $\rho \vec{u}$ and momentum-flux tensor $\Pi$. Hence, the regularization filters 
out non-hydrodynamics modes from the non-equilibrium component of the postcollision distribution functions, thus preserving the non-equilibrium component of the distribution from ghost contamination.

The choice of the regularization procedure is motivated by the fact that, in a previous work ([19]) it has been shown that a standard LB enriched with regularization procedure and Higher Order Lattices (HOL) allows to quantitatively predict mass flow in porous media across the full range of Knudsen numbers (from hydro to ballistic regime). In the same paper, it is also shown that, standard LB equipped with regularization step provides reasonable results both in the slip and transition regimes $(K n \leq 1)$. This fact can be explained by noting that, for the Kn numbers at hand, even without HOL, the artifacts due to runaway directions( i.e. the tendency of the LB lattices to overemphasize the role of the streaming directions which never hit the wall in the collisionless limit) are substantially suppressed by regularization. Doubtless, the use of HOL regularized models is needed to account for non-equilibrium effects at high Knudsen numbers so as to accomplish the so called Grad's extended hydrodynamics but, at least for the problem studied in this paper, where the non-equilibrium effects seems not to play a dramatic role as shown in [19], the low order regularized model still perform reliably. For the sake of clarity, here we report a pseudocode of the regularization procedure employed in our simulations (eqn.).

$$
\begin{aligned}
& \text { for } l \leq b \wedge \forall(i, j, k) \in D \text { do } \\
& \qquad f_{l}^{n e q}(i, j, k)=f_{l}^{p c}(i, j, k)-f_{l}^{e q}(i, j, k)
\end{aligned}
$$

end for

$$
\begin{aligned}
& \text { for } l \leq b \wedge \forall(i, j, k) \in D \text { do } \\
& \qquad p_{\alpha \beta}(i, j, k)=p_{\alpha \beta}(i, j, k)+\left(c_{l \alpha} c_{l \beta}-c_{s}^{2} \delta_{\alpha \beta}\right) f_{l}^{n e q}(i, j, k) \\
& \text { end for } \\
& \text { for } l \leq b \wedge \forall(i, j, k) \in D \text { do } \\
& \qquad f_{l}^{r e g}(i, j, k)=f_{l}^{e q}(i, j, k)+\sum_{l} \frac{w_{l}}{2 c_{s}^{4}}\left(c_{l \alpha} c_{l \beta}-c_{s}^{2}\right) p_{\alpha \beta}(i, j, k)
\end{aligned}
$$

end for 
where $f_{l}^{p c}(i, j, k)$ is the set of post-collision distribution functions, $f_{l}^{n e q}(i, j, k)$ is the non equilibrium part of $f_{l}^{p c}(i, j, k), p_{\alpha \beta}$ are the components of the nonequilibrium part of the momentum flux tensor, $D$ stands for the fluid domain and $f_{l}^{r e g}(i, j, k)$ is the regularized set of post-collision distribution functions.

\subsection{The reactive boundary condition}

In this paper, we employ the standard version of the LBM for a threecomponent system, consisting of an inert carrier $(\mathrm{C})$, a reactant $(\mathrm{R})$ and product (P) species. Chemical reactions are patterned after the following methanol oxidation reaction [23],

$$
2 \mathrm{CH}_{3} \mathrm{OH}+\mathrm{O}_{2} \rightarrow \mathrm{HCOOCH}_{3}+2 \mathrm{H}_{2} \mathrm{O},
$$

Species interconversion due to catalytic reactions at the pore surface is accounted for by considering a local exchange of populations as they meet and react on the solid walls of the pore. For the case of heterogeneous catalysis, such reaction takes places whenever gas populations hit the surface of the porous catalyst. Here, we consider a first order chemical reaction of the generic form $\mathrm{R} \rightarrow \mathrm{P}$.

The reactive boundary condition is designed such that, upon colliding with a solid site, the reactant $\mathrm{R}$ converts into the product $\mathrm{P}$ with a given probability $p$. Thus, each molecule of $\mathrm{R}$ colliding with a catalytic site is re-emitted along a random direction, either as species $\mathrm{R}$ with probability $1-p$ (no-reaction), or as species $\mathrm{P}$ with probability $p$. It is worth highlighting that, in order to fulfill mass conservation locally, $p$ is uniform in the domain and constant in time. Within this framework a typical chemical timescale can be defined as $\tau_{c h}=\Delta t / p=1 / p$ (in lattice units), which is the time interval needed for one representative molecule of product to enter the system (i.e. to be produced). First order surface chemical reactions have been modelled by implementing a "sputtering" boundary condition, as depicted in Figure 1. In equations, 


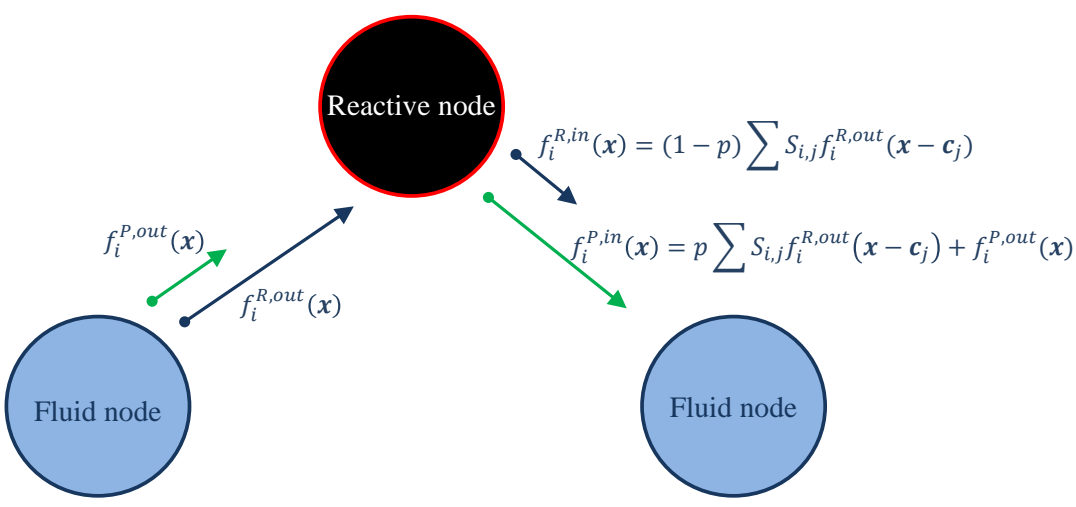

Figure 1: The sputtering reactive boundary condition

$$
\begin{aligned}
f_{i}^{R, \text { in }}(\boldsymbol{x}) & =(1-p) \sum_{j=1}^{b(\boldsymbol{x})} S_{i, j} f_{i}^{R, \text { out }}\left(\boldsymbol{x}-\boldsymbol{c}_{j}\right) \\
f_{i}^{P, \text { in }}(\boldsymbol{x}) & =p \sum_{j=1}^{b(\boldsymbol{x})} S_{i, j} f_{i}^{R, \text { out }}\left(\boldsymbol{x}-\boldsymbol{c}_{j}\right)+f_{i}^{P, \text { out }}(\boldsymbol{x})
\end{aligned}
$$

where $S_{i, j}$ is a random sputtering matrix obeying the mass conservation constraint, $\sum_{j=1}^{b(\boldsymbol{x})} S_{i, j}=1$ and $0 \leq b(\boldsymbol{x}) \leq 18$ is the number of active links at any given lattice site $\boldsymbol{x}$. According to this model, each node of the porous medium in direct contact with the gas nodes acts as a catalytic site. The boundary condition is built in such a way as to locally preserve the $\mathrm{R}+\mathrm{P}$ mass, while the $\mathrm{C}$ mass is conserved anyway since the carrier does not take part to the reaction. The random, although discrete, orientation of the emerging populations mimics the microscopic effect of the wall orientation on the gas dynamics and the fact that the particles that are adsorbed by the catalyst have sufficient time to loose memory of their incoming directions thus making the scattering angles effectively random. In the present boundary condition (see Fig. 1) such randomization mechanism acts independently on the two species ( $R$ and $P$ ), while the carrier is subject to bounce-back boundary rule which have been shown to capture lumped quantities (say mass flow) in finite-Knudsen flows, once combined with Regularization (see [19]). 


\section{Relevant non-dimensional numbers}

The main governing dimensionless parameters of reactive fluid transport are: the Reynolds number $R e=U d / \nu$, measuring inertial versus dissipative effects, the Mach number $M a=U / c_{s}$, fluid versus sound speed and the Knudsen $K n=\nu / c_{s} d$, namely the molecular mean free path versus the average flow characteristic length scale. A second group is composed by the Peclét $P e=U d / D$ (advection over diffusion), advective Damköhler $D a_{A}=c_{s} p / U$ (chemical versus advection timescales), diffusive Damköhler $D a_{D}=c_{s} p d / D$, (chemical versus diffusive timescale) and Schmidt $S c=\nu / D$ (momentum versus mass diffusion). The following notation is employed: $U$ and $d$ are respectively the characteristic pore velocity and spacing, $\nu$ is the fluid kinematic viscosity and $D$ is the diffusivity of the chemical species.

It is possible to identify five quantities describing the physical system, namely, $U, \nu, D, d, c_{s}$ involving two physical dimensions,length and time. A straightforward application of the $\Pi$ theorem allows to formulate three independent nondimensional groups. Therefore, the following relations hold:

$$
K n=M a / R e \quad P e=S c R e \quad D a_{D}=P e D a_{A}
$$

Given the three relations (6), one is left with four independent parameters, $K n$, $D a_{D}, P e, S c$. When the pore thickness is small compared to the mean-free path of the molecules, say $K n>1$, molecules collide with the pore surface with higher probability than bulk intermolecular collisions. The resulting total diffusivity, namely $\frac{1}{D_{\text {tot }}}=\frac{1}{D_{M}}+\frac{1}{D_{K}}$ gets lower [1] where $D_{M}$ and $D_{K}$ are the molecular and Knudsen diffusion coefficients, respectively. The investigation of the dependence on the $K n$ is here restricted to $P e=O\left(10^{-5}\right)$, as it pertains to rarefied gas flows in catalytic media.

\section{Model validation}

The model has been first validated against Lévêque's analytical solution [31] of a two-dimensional laminar reactive flow between two parallel plates, reactions 


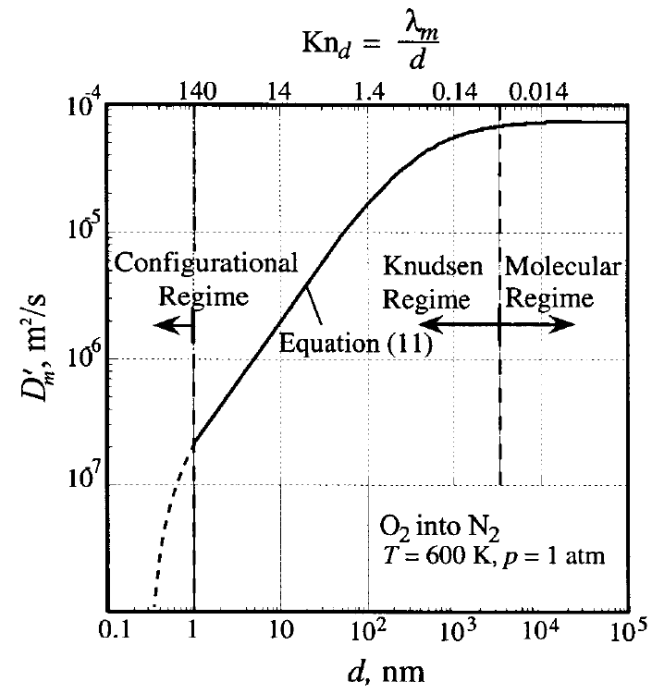

Figure 2: Dependence of the total diffusivity on pore diameter or, given a mean free molecular path, on $K n$. In this figure "Molecular Regime" denotes the low-Knudsen collective particle (hydrodynamic) regime, whereas "Configurational Regime" stands for high-Knudsen freeparticle motion. Note that Knudsen increases from right to left. From [1]

taking place at one of the two. The vertical gradient of concentration at the reactive wall can be calculated as:

$$
\frac{\partial C_{R}}{\partial y}(x)=\frac{1}{\Gamma(4 / 3) 9^{1 / 3}}\left(\frac{4 P e}{x / L_{y}}\right)^{1 / 3}
$$

where $C_{R}(x)$ is the reactant concentration distribution along the reactive wall, $\Gamma$ is the Gamma function [32], $x$ is the streamwise direction and $L_{y}$ is the distance between plates. As per the Peclét number, Levesque's solution is valid for high $P e$. According to this, the Peclét number was set to $P e \simeq 253$.

The benchmark test was carried out on a $150(x) \times 50(y)$ grid. A body force has been used to simulate the constant pressure gradient along the channel. The reactant is injected continuously at the inlet of the channel and reacts only with the lower plate. The reaction probability in the sputtering boundary conditions has been set to 1 , so as to simulate instantaneous reaction, $\left(D a_{A} \rightarrow \infty\right)$. In the limit of infinite $D a_{A}$, all the reactant impacting on the active nodes are 


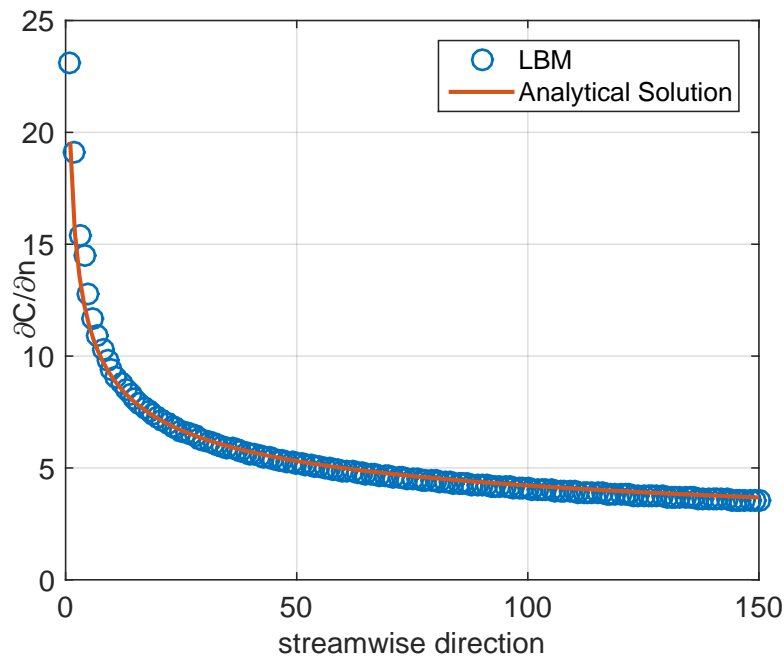

Figure 3: Comparison between analytical solution of the 2 d laminar reactive flow between parallel plates. The concentration gradient along the crossflow direction at the reaction plate is plotted against the streamwise direction.

instantly converted into products. Good agreement with the analytical solution is found, as evidenced by the comparison reported in Figure 3. The overall Mean Absolute Error, is roughly 4\%.

\section{Numerical simulations}

Simulations have been carried out on a numerical sample of nanoporous medium, with a resolution of $128^{3}$ nodes and a porosity of $70 \%$, with random pore distribution. The value of the porosity is chosen so as to match that of the nano-porous gold ingot used in the experiments. As per the grain dimension, each grain is discretized with ten lattice spacings on average. Flow inside the medium has been obtained by applying a constant mass force to the carrier distribution. Boundary conditions are as follows:

- carrier: periodic boundary conditions at all domain boundaries;

- reactant: continuous constant injection of unit concentration at inlet, zerogradient at oultet, periodic for crossflow directions; 


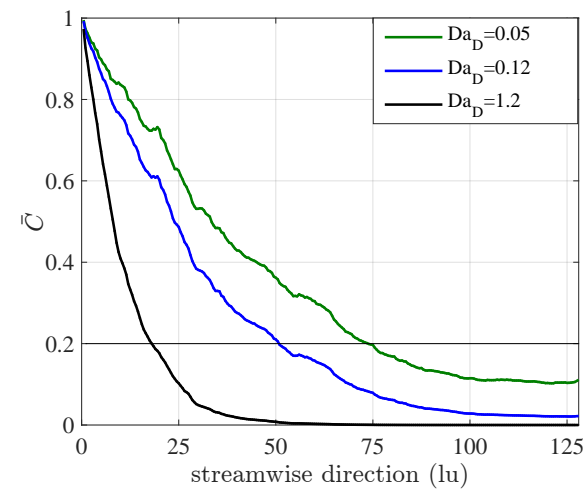

(a)

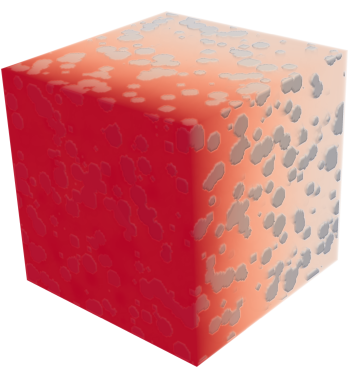

(b)

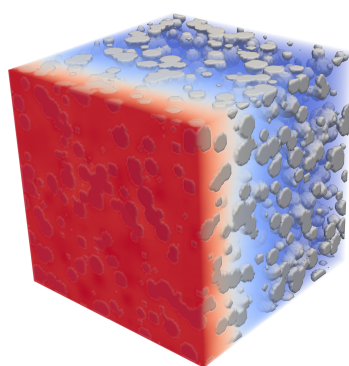

(c)

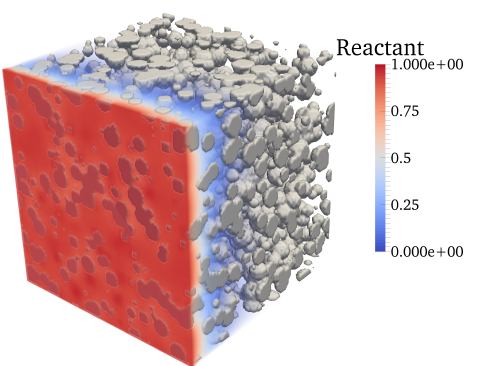

(d)

Figure 4: Reactant concentration across the sample for three different $D a_{D}$ namely, (a) $D a_{D}=$ 0.05 , (b) $D a_{D}=0.12$ and (c) $D a_{D}=1.2$. Thick line at the $20 \%$ value identifies the assumed penetration threshold.

- reactant: zero-gradient at inlet and outlet; periodic for crossflow directions;

The simulations are advanced in time until the flow and concentration fields reached the steady state. Several numerical simulations have been carried out by varying $D a_{D}$ and $K n$ numbers independently. The results are analyzed in terms of Depth of Penetration (DoP) of the reactant, which we define as the distance from the inlet section at which $80 \%$ of the Reactant is consumed, that is where the average concentration on a crossflow section of the specimen, namely $\bar{C}$, falls below $20 \%$. The reactant concentration hereinafter is normalized to the inlet reactant concentration. 


\subsection{Effect of the Damköhler number}

In this subsection, we study the effect of Damköhler number. Peclét number is chosen of order $O\left(10^{-5}\right)$ in all simulations, for matter of consistency with the experimental flow conditions within the ingot nano-pores previously mentioned. Indeed, with reference to the experimental facility described in [22], by taking a typical flow rate through the reactor of the order of $50 \mathrm{~mL} / \mathrm{min}$ and a pipe diameter $=25 \mathrm{~mm}$, the outside velocity can be estimated of the order $0.001 \mathrm{~m} / \mathrm{s}$. The effective diffusion coefficient inside the pores is estimated as $\simeq 10^{-6} \mathrm{~m}^{2} / \mathrm{s}$. The pore characteristic diameter $d$ is $\approx 50 \mathrm{~nm}$; inside such pores, the actual flow speed is expected to be, at least, one or two order of magnitude lower than the outside flow velocity. This said, a Peclét number of $O\left(10^{-5}\right)$ is an appropriate choice, resulting in a gas speed $10^{-4} \mathrm{~m} / \mathrm{s}$ inside the pore. From Fig.4 (a), the major effect of $D a_{D}$ on the depth of penetration is apparent. The lower the Damköhler, the deeper the penetration of the reactant into the bulk of the nano-porous catalyst. This is very intuitive, but the present simulations provide the effective penetration depth in quantitative terms.

\subsection{Effect of the Knudsen number}

The procedure followed to carry out the simulations is here outlined: the scalar relaxation time defines $D_{M}$, and consequently it is used to define the diffusive Damkohler number, $D a_{D}$, reported in Figure 6 . Throughout the work molecular diffusivity was assumed to be equal to that of the product. The Knudsen number is varied by acting on kinematic viscosity, through the relaxation rate of the carrier. It is worth recalling that at high $K n$ numbers, the total diffusivity, $D_{t o t}$, receives a further contribution from $D_{K}$. With respect to Fig. 5 , the higher the $D_{K}$ the higher the $D_{t o t}$, but $D_{\text {tot }}$ reduces to $D_{M}$ for $D_{K} \rightarrow \infty$. Since $D_{K} \propto d \propto \lambda / K n$ then $D_{K} \propto 1 / K n$, the DoP automatically accounts for the effect of the total diffusivity, which is a decreasing function of the $K n$. Here, we analyze the DoP at two different $K n(0.1$ and 1$)$ and three different $D a_{D}$ $(0.05,0.12$ and 1.2). In Fig. 6 the $\bar{C}$ profiles measure the coupled effect of the $K n$ and $D a_{D}$. Firstly, the simulations confirm the decreasing trend of the DoP 


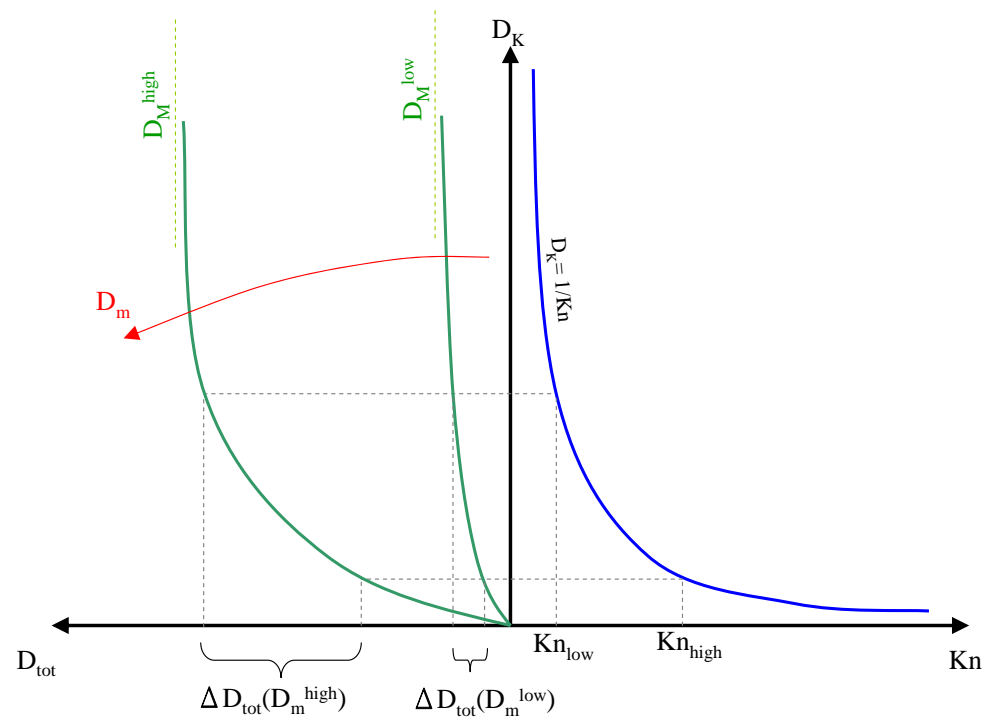

Figure 5: Qualitative sketch showing the dependency of the $D_{t o t}$ on the $K n$ through the $D_{K}$. The higher the $K n$ the higher the $D_{t o t}$, but effect is strongly mitigated by the value of $D_{M}$, that is, the higher the $D_{M}$ the larger the gain in $D_{t o t}$. Note that, for $K n \rightarrow 0$, (hydrodynamic regime), $D_{K} \rightarrow \infty D_{\text {tot }} \rightarrow D_{M}$.

at increasing $D a_{D}$ for each different Knudsen number. In agreement with the theoretical predictions shown in Fig. 5 our results show a decreasing trend in the DoP as $K n$ increases, for a given $D a_{D}$. More interestingly, Fig.6 highlights that the effects of $K n$ are mitigated at higher values of $D a_{D}$ (which means lower values of $D_{M}$ ), which is in compliance with the theoretical prediction sketched in Fig. 5. This means that the higher the molecular diffusion of the gas, the higher the sensitivity to a variation of the pore diameter.

\subsection{Towards large-scale simulations}

An all-encompassing full-scale simulation of nanocatalytic devices is clearly beyond the capabilities of present-day most powerful computers, and likely to remain such for many years to come. Under such state of affairs, it is imperative to develop upscale strategies whereby the microscale details are conveyed into effective meso/macroscale parameters, to be used in large scale simulations at affordable resolution. For the problem in point, it is of great relevance to develop 


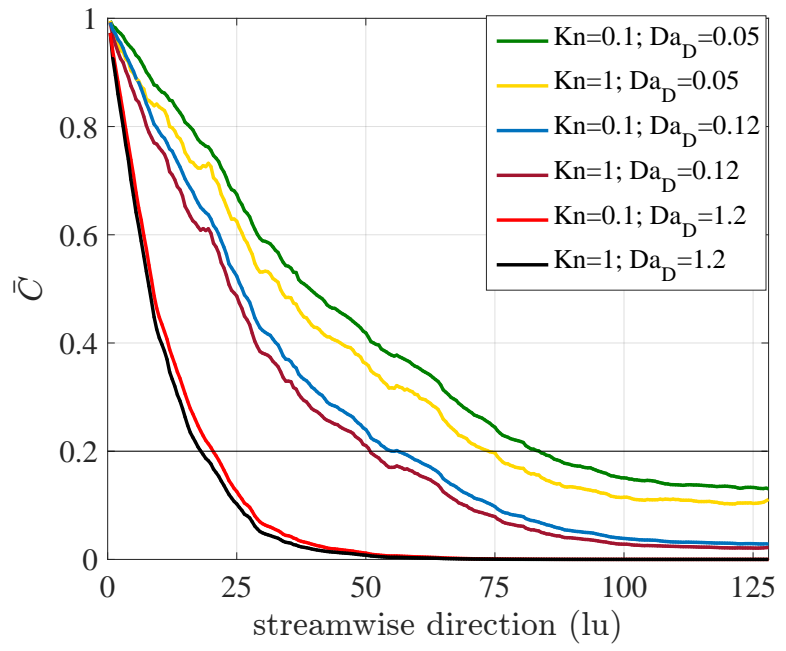

Figure 6: Combined effect of $D a_{D}$ and $K n$ on the steady state distribution of $\bar{C}$ along the streamwise direction.

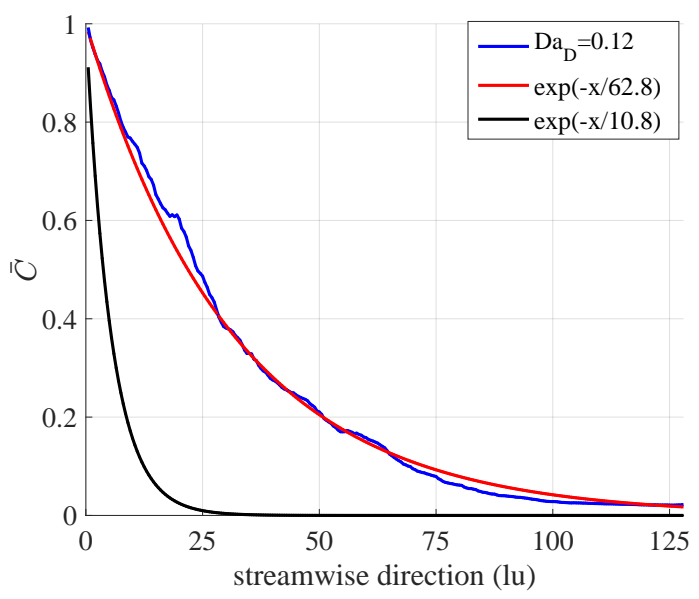

Figure 7: Best fit of the numerical solution gives delta $=62.8$, i.e. about six times larger than the corresponding value for the homogeneous media. This reflects the reduced reactivity due to the porous structure of the material. 
a quantitative assessment of the typical lengthscale at which chemical reactivity occurs within the nanoporous sample. To this purpose, we compared the numerical LBM solution with the analytical solution of a steady one-dimensional homogeneous reactive medium, fed at a constant rate of reactant and governed by the following equation [33]:

$$
U \frac{\partial C}{\partial x}=D \frac{\partial^{2} C}{\partial x^{2}}+\frac{C}{\tau_{c h}}
$$

Equation (8) can be cast in non-dimensional form:

$$
P e \frac{\partial C}{\partial x^{*}}=\frac{\partial^{2} C}{\partial x^{* 2}}+D a_{D} C
$$

where $x^{*}=x / L$ is the dimensionless reactive length. The solution of eq. 9 reads as: $C\left(x^{*}\right)=\exp \left(-x^{*} / \delta\right)$, where $\delta=\left[1 / 2\left(P e-\sqrt{P e^{2}-4 D a_{D}}\right)\right]^{-1}$ is the characteristic non-dimensional length scale of the homogeneous reactive layer. In Fig. 7 , the numerical solution is plotted together with the analytical aforementioned solution for the homogeneous medium, as computed with the values of $P e$ and $D a_{D}$ pertaining to the simulation. First of all, it is worth highlighting that the reactant concentration still yields the classical exponential decay also at finite Knudsen numbers. As this figure also makes apparent, the idealised homogeneous medium is significantly more efficient, i.e. it yields a shorter reactive layer than the nanoprous sample. In an upscaling perspective, one determines $\delta$ as the best fit to the analytical solution versus the numerical one. Such procedure yields the concentration distribution shown in Fig. 7, from which it is seen that the effective reactive length in the nanoporous sample is about six times longer than in the homogeneous material. This assessment provides the first step to large-scale simulation of catalytic devices of engineering relevance, with coarsemesh spacings of the order $\Delta x \sim \delta$, rather than $\Delta x<<\delta$, as it is the case in micro-resolved simulations such as the one presented in the current work. 


\section{Summary and Outlook}

Summarizing, we have investigated the effects of hydrodynamic non-equilibrium, as measured by the Knudsen number, on the reactivity of nanoporous catalytic samples. Extensive numerical simulations indicate that non-equilibrium effects enhance the effective reactivity of the sample (see Section 5). Comparison with a minimal 1D model shows that the streamwise distribution of reactant concentration still yields the classical exponential decay also at finite Knudsen numbers, as shown in subsections 5.2 and 5.3. This provides a first step towards the inclusion of the reactive and diffusive properties of the medium beyond the hydrodynamic regimes within large-scale simulations of catalytic devices of experimental relevance.

\section{Acknowledgments}

This work was partly supported by the Integrated Mesoscale Architectures for Sustainable Catalysis(IMASC), an Energy Frontier Research Center funded by the US Department of Energy, Office of Science, Basic Energy Sciences under Award No. DE-SC0012573.

\section{References}

[1] M. M. Mezedur, M. Kaviany, W. Moore, Effect of pore structure, randomness and size on effective mass diffusivity, AIChE journal 48 (1) (2002) $15-24$.

[2] E. Jannelli, M. Minutillo, A. L. Lavadera, G. Falcucci, A small-scale caes (compressed air energy storage) system for stand-alone renewable energy power plant for a radio base station: A sizing-design methodology, Energy 78 (2014) 313-322.

[3] G. Falcucci, E. Jannelli, M. Minutillo, S. Ubertini, J. Han, S. Yoon, S. Nam, Integrated numerical and experimental study of a mcfc-plasma gasifier energy system, Applied Energy 97 (2012) 734-742. 
[4] F. Higuera, S. Succi, R. Benzi, Lattice gas dynamics with enhanced collisions, Europhysics Letters 9 (4) (1989) 345.

[5] R. Benzi, S. Succi, M. Vergassola, The lattice boltzmann equation: theory and applications, Physics Reports 222 (3) (1992) 145-197.

[6] S. Succi, The lattice Boltzmann equation: for fluid dynamics and beyond, Oxford university press, 2001.

[7] S. Succi, E. Foti, F. Higuera, Three-dimensional flows in complex geometries with the lattice boltzmann method, EPL (Europhysics Letters) 10 (5) (1989) 433.

[8] B. Ferreol, D. H. Rothman, Lattice-boltzmann simulations of flow through fontainebleau sandstone, in: Multiphase Flow in Porous Media, Springer, 1995, pp. 3-20.

[9] N. S. Martys, H. Chen, Simulation of multicomponent fluids in complex three-dimensional geometries by the lattice boltzmann method, Physical Review E 53 (1) (1996) 743.

[10] Z. Guo, T. Zhao, Lattice boltzmann model for incompressible flows through porous media, Physical Review E 66 (3) (2002) 036304.

[11] M. E. Kutay, A. H. Aydilek, E. Masad, Laboratory validation of lattice boltzmann method for modeling pore-scale flow in granular materials, Computers and Geotechnics 33 (8) (2006) 381-395.

[12] O. Aursjø, G. Løvoll, H. A. Knudsen, E. G. Flekkøy, K. J. Måløy, A direct comparison between a slow pore scale drainage experiment and a $2 \mathrm{~d}$ lattice boltzmann simulation, Transport in porous media 86 (1) (2011) 125-134.

[13] A. E. Khabbazi, J. Ellis, A. Bazylak, Developing a new form of the kozenycarman parameter for structured porous media through lattice-boltzmann modeling, Computers \& Fluids 75 (2013) 35-41. 
[14] M. C. Sukop, H. Huang, P. F. Alvarez, E. A. Variano, K. J. Cunningham, Evaluation of permeability and non-darcy flow in vuggy macroporous limestone aquifer samples with lattice boltzmann methods, Water Resources Research 49 (1) (2013) 216-230.

[15] D. Brown, J. Li, M. Ghommem, V. Calo, Upscaling of lattice boltzmann simulations in heterogeneous media and related reduced order modelling techniques., Mathematical Modeling of Flow and Transport in Porous Media 19.

[16] H. Daigle, J. S. Reece, Permeability of two-component granular materials, Transport in Porous Media 106 (3) (2015) 523-544.

[17] A. Cali, S. Succi, A. Cancelliere, R. Benzi, M. Gramignani, Diffusion and hydrodynamic dispersion with the lattice boltzmann method, Physical Review A 45 (8) (1992) 5771.

[18] P. Prestininzi, A. Montessori, M. La Rocca, S. Succi, Reassessing the single relaxation time lattice boltzmann method for the simulation of darcy's flows, International Journal of Modern Physics C (2015) 1650037.

[19] A. Montessori, P. Prestininzi, M. La Rocca, S. Succi, Lattice boltzmann approach for complex nonequilibrium flows, Physical Review E 92 (4) (2015) 043308 .

[20] A. Montessori, G. Falcucci, M. La Rocca, S. Ansumali, S. Succi, Threedimensional lattice pseudo-potentials for multiphase flow simulations at high density ratios, Journal of Statistical Physics (2015) 1-16.

[21] G. Falcucci, G. Bella, G. Chiatti, S. Chibbaro, M. Sbragaglia, S. Succi, Lattice boltzmann models with mid-range interactions, Communications in Computational physics 2 (2007) 1071-1084.

[22] L.-C. Wang, K. J. Stowers, B. Zugic, M. L. Personick, M. M. Biener, J. Biener, C. M. Friend, R. J. Madix, Exploiting basic principles to control the 
selectivity of the vapor phase catalytic oxidative cross-coupling of primary alcohols over nanoporous gold catalysts, Journal of Catalysis 329 (2015) $78-86$.

[23] M. L. Personick, B. Zugic, M. M. Biener, J. Biener, R. J. Madix, C. M. Friend, Ozone-activated nanoporous gold: A stable and storable material for catalytic oxidation, ACS Catalysis.

[24] C. Aidun, J. Clausen, Lattice-boltzmann method for complex flows, Annual Review of Fluid Mechanics 42 (2010) 439-472.

[25] S. Chen, G. Doolen, Lattice boltzmann method for fluid flows, Annual review of fluid mechanics 30 (1) (1998) 329-364.

[26] J. Latt, B. Chopard, Lattice boltzmann method with regularized precollision distribution functions, Mathematics and Computers in Simulation 72 (2) (2006) 165-168.

[27] R. Zhang, X. Shan, H. Chen, Efficient kinetic method for fluid simulation beyond the navier-stokes equation, Physical Review E 74 (4) (2006) 046703.

[28] A. Montessori, G. Falcucci, P. Prestininzi, M. La Rocca, S. Succi, Regularized lattice bhatnagar-gross-krook model for two-and three-dimensional cavity flow simulations, Physical Review E 89 (5) (2014) 053317.

[29] X.-D. Niu, S.-A. Hyodo, T. Munekata, K. Suga, Kinetic lattice boltzmann method for microscale gas flows: issues on boundary condition, relaxation time, and regularization, Physical Review E 76 (3) (2007) 036711.

[30] H. Chen, R. Zhang, I. Staroselsky, M. Jhon, Recovery of full rotational invariance in lattice boltzmann formulations for high knudsen number flows, Physica A: Statistical Mechanics and its Applications 362 (1) (2006) 125131.

[31] A. Lévêque, Les Lois de la transmission de chaleur par convection, par André Lévêque, Dunod, 1928. 
[32] M. Abramowitz, I. A. Stegun, Handbook of mathematical functions: with formulas, graphs, and mathematical tables, no. 55, Courier Corporation, 1964.

[33] S. Socolofsky, G. Jirka, Environmental fluid mechanics 1: Mixing and transport processes in the environment, Texas A and M University. 